\title{
Alcohol Flushing and Positive Ethanol Patch Test in Patients with Coronary Spastic Angina: Possible Role of Aldehyde Dehydrogenase 2 Polymorphisms
}

\author{
Yuji Mizuno ${ }^{1}$, Sumio Morita ${ }^{1,2}$, Eisaku Harada ${ }^{1}$, Makoto Shono ${ }^{1}$, \\ Yoshinobu Morikawa ${ }^{3}$, Toyoaki Murohara ${ }^{2}$ and Hirofumi Yasue ${ }^{1}$
}

\begin{abstract}
Objective Coronary spasm plays an important role in the pathogenesis of coronary heart disease (CHD) and angina pectoris caused by coronary spasm or coronary spastic angina (CSA) is prevalent in Japan. However, the precise mechanisms underlying coronary spasm are unclear. Alcohol intolerance is prevalent among East Asians, and we previously reported that coronary spasm could be induced by alcohol intake in CSA patients. We herein examined whether CSA is associated with alcohol intolerance in Japanese subjects.

Methods The study subjects consisted of $80 \mathrm{CSA}$ patients $(57 \mathrm{men} / 23$ women, mean age $62 \pm 12)$ and 52 non-CSA patients $(25 \mathrm{men} / 27$ women, mean age $63 \pm 10)$. The ethanol patch test (EPT) and questionnaire which evaluates flushing after ethanol intake, along with an examination of clinical features and laboratory chemistry data for CHD risk factors were done. Gender (male) and smoking were higher $(\mathrm{p}=0.007$, and $\mathrm{p}=$ 0.019 , respectively) and plasma HDL cholesterol level was lower $(\mathrm{p}=0.035)$ in the CSA patients than in the non-CSA patients. Multivariable logistic regression analysis including age, EPT, smoking, and plasma HDL cholesterol level as independent variables revealed that positive EPT and smoking were significant predictors of CSA ( $\mathrm{p}=0.011$ and $\mathrm{p}=0.016$, respectively).

Conclusion Positive EPT and alcohol flushing following alcohol intake, as well as smoking and plasma levels of HDL cholesterol, were significantly associated with CSA in Japanese patients. Therefore, alcohol ingestion as well as smoking is a significant risk factor for CSA in Japanese.
\end{abstract}

Key words: alcohol, acetaldehyde dehydrogenase 2, coronary spastic angina, ethanol patch test, smoking

(Intern Med 52: 2593-2598, 2013)

(DOI: 10.2169/internalmedicine.52.0894)

\section{Introduction}

Coronary artery spasm plays an important role in the pathogenesis of coronary heart disease (CHD), including unstable angina, acute myocardial infarction and sudden cardiac death $(1,2)$. Coronary spastic angina (CSA) or angina pectoris caused by coronary artery spasm is prevalent among the East Asian populations, including Japanese (1-3). Ethanol is metabolized first to acetaldehyde by alcohol dehydrogenase $(\mathrm{ADH})$ and then to acetic acid by acetaldehyde dehydrogenase 2 (ALDH2) (4). East Asians have been shown to have a mutation of the ALDH2*2 gene that leads to a reduced activity and intolerance to alcohol (ethanol) ingestion in about half of the population (5-8). We and others have reported that CSA is induced by alcohol ingestion, particularly in those who have alcohol flushing upon alcohol intake (9-11). However, the relationship between alcohol intake and CSA has not been elucidated. Recent studies have shown that the ethanol (alcohol) patch test (EPT) is a sensitive marker of the Asian type of ALDH2*2 polymorphism (12-14). We designed the present study to examine the relationship between the $\mathrm{ALDH} 2 * 2$ polymorphism and CSA by using the EPT and questionnaires on alcohol intake.

${ }^{1}$ Division of Cardiovascular Medicine, Kumamoto Kinoh Hospital, Kumamoto Aging Research Institute, Japan, ${ }^{2}$ Department of Cardiology, Nagoya University Postgraduate School of Medicine, Japan and ${ }^{3}$ Division of Cardiovascular Medicine, Nara City Hospital, Japan Received for publication April 30, 2013; Accepted for publication June 24, 2013 Correspondence to Dr. Yuji Mizuno, mizuno@juryo.or.jp 


\section{Materials and Methods}

\section{Study subjects}

The study subjects consisted of 132 patients ( 82 men with a mean age of $66.3 \pm 10.9$, and 50 women with a mean age of $67.4 \pm 10.0$ ) who were admitted to our institution between January, 2010 and April 2013, and in whom coronary angiography and intracoronary injection of acetylcholine (ACh) were performed on suspicion of coronary spastic angina. Eighty patients (57 men and 23 women, mean age 62 \pm 12 ) were diagnosed as CSA on the basis of angiographically documented coronary spasm and the remaining 52 patients

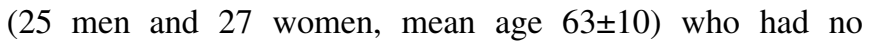
coronary spasm induced served as the controls. Patients with acute myocardial infarction, organic stenosis of $\geq 90 \%$, multivessel organic disease, left main trunk lesion, myocardial infarction, uncontrolled arrhythmias, heart failure, resting hypertension $>180 / 110 \mathrm{mmHg}$, acute systemic illness, hepatic or renal insufficiency or other severe conditions were excluded from the study. All vasoactive medications including calcium channel blockers, beta-receptor blockers, angiotensin converting enzyme inhibitors, and angiotensin II receptors blockers were withdrawn for at least three days before performing angiography except for nitroglycerin used for attacks. The study was approved by the ethics committee of our institution and written informed consent was obtained from each patient.

\section{Angiographic documentation of coronary spasm}

All anti-angina drugs were discontinued for at least 72 hours except for sublingual nitroglycerin, which was also withdrawn eight hours before coronary angiography. Coronary spasm was defined as an abnormal contraction of an epicardial coronary artery associated with transient ischemic changes on electrocardiogram (ECG) and chest discomfort. Coronary spasm was documented by the intracoronary injection of ACh (Daiichi-Sankyo Co., Tokyo, Japan) after diagnostic catheterization in the morning. The details of the method have been reported previously (15). Briefly, ACh was injected in incremental doses of 20,50 and $100 \mu \mathrm{g}$ into the left coronary artery, and then 20 and, $50 \mu \mathrm{g}$ into the right coronary artery under continuous monitoring of 12 lead ECG and blood pressure. Finally, nitroglycerin or isosorbide dinitrate was infused to relieve spasm and examine organic lesions. Significant organic coronary stenosis was defined $>50 \%$ luminal diameter and coronary artery disease was divided into one vessel, two vessel and three vessel disease.

\section{Ethanol patch test}

All patients refrained from the ingestion of alcohol for at least 12 hours before the EPT. The test was performed using a lucent patch of water-containing gel containing $14 \%$ ethanol attached to polyolefin-film that measured $11 \mathrm{~mm} \times 11$ $\mathrm{mm} \times 100 \mu \mathrm{m}$ (Alcohol Constitution Test Patch, Life Care Giken Co., Toyama, Japan) (16). The patch was attached to the inner surface of the upper arm for 20 minutes and then removed. A patch area showing erythema after removal was judged to be positive. The judgment was made by one of the medical staff blinded to the results of the coronary angiograms and questionnaire.

\section{Questionnaire survey}

The subjects were asked to fill out a simple questionnaire concerning alcohol flushing on alcohol intake, alcohol drinking habit, and smoking. The flushing questions consisted of "always flushing", "former or sometimes flushing", and "never flushing". "Always flushing" and "former or sometimes flushing" combined were designated as positive and "never flushing" as negative for flushing. Smokers were defined as current and former smokers.

\section{Blood chemistry measurements}

Blood samples for measurement of clinical chemistry and other data were collected after an overnight fast with the patients in the supine position. The biochemical and other analyses were done using standard laboratory procedures.

\section{Statistical analysis}

The data were expressed as the mean \pm SD or median $(25$ th, 75th percentile) for continuous variables and differences within the group were evaluated with unpaired t-test or the Mann-Whitney rank sum test. For discrete variables, the data were expressed as counts and percentages and analyzed with the Chi square test. The correlation between the variables was assessed using Spearman's rank correlation coefficient. Variables that were not statistically significant (i.e., p> $0.05)$ were excluded from further analyses. A multiple logistic regression analysis was performed to determine the contribution of various clinical variables to CSA. Predictor variables were included on the basis of theoretical grounds and the results of a bivariate analysis. A two-tailed value of $\mathrm{p}<$ 0.05 was considered to be as statistically significant. The analyses were done using the STATA software program (STATA 11.0, STATA Corp., College Station, TX, U.S.A.).

\section{Results}

Sixty five of the $80(81.3 \%)$ CSA patients and 46 of the $52(88.5 \%)$ non-CSA patients had no significant organic coronary stenosis. Thus, the majority of the study subjects had no significant organic stenosis in the coronary arteries, and there was no significant difference in the prevalence of organic stenosis between the two groups.

Table 1 shows the clinical characteristics of the CSA and non-CSA groups. There were no significant differences in the age, body mass index, blood pressure, or plasma levels of albumin, LDL cholesterol, triglycerides, blood glucose, and C-reactive protein between the two groups. However, male gender, smoker and plasma uric acid levels were sig- 
Table 1. A Comparison of the Clinical Characteristics between the CSA and Non-CSA Groups

\begin{tabular}{lccc}
\hline Variables & Non-CSA $(\mathrm{n}=52)$ & CSA $(\mathrm{n}=80)$ & p value \\
\hline Age, years & $66.3 \pm 10.9$ & $67.4 \pm 10.0$ & 0.561 \\
Men, $\mathrm{n}(\%)$ & $25(48.1)$ & $57(71.3)$ & 0.007 \\
BMI, $\mathrm{kg} / \mathrm{m}^{2}$ & $23.5 \pm 4.1$ & $24.4 \pm 7.1$ & 0.397 \\
Systolic BP, mmHg & $132.2 \pm 21.0$ & $129.9 \pm 17.6$ & 0.507 \\
Diastolic BP, mmHg & $77.0 \pm 11.4$ & $73.9 \pm 12.3$ & 0.147 \\
Albumin, g/dL & $4.1(3.9,4.3)$ & $4.0(3.8,4.2)$ & 0.102 \\
Hs-CRP, $\mu \mathrm{g} / \mathrm{L}$ & $470(250,9575)$ & $760(305,21525)$ & 0.123 \\
Glucose, mg/dL & $101(92,119.5)$ & $95.3(87.0,102)$ & 0.51 \\
Total cholesterol, mg/dL & $190(167,218)$ & $196(161,218)$ & 0.817 \\
Triglycerides, mg/dL & $117(85,155)$ & $123(86,179)$ & 0.582 \\
HDL-C, mg/dL & $57(47,76)$ & $51(43.3,67)$ & 0.035 \\
LDL-C, mg/dL & $104(74,125)$ & $110(87,126)$ & 0.182 \\
AST, U/L & $28.9 \pm 18.9$ & $27.5 \pm 12.1$ & 0.608 \\
ALT, U/L & $22.6 \pm 13.1$ & $21.7 \pm 11.2$ & 0.661 \\
Uric acid, mg/dL & $4.8 \pm 1.2$ & $5.5 \pm 1.3$ & 0.007 \\
e-GFR, mL/min./1.73m ${ }^{2}$ & $70.3 \pm 17.5$ & $67.8 \pm 15.1$ & 0.385 \\
Smokers, $\mathrm{n}(\%)$ & $23(44.2)$ & $52(65.0)$ & 0.019 \\
Alcohol flushing, $\mathrm{n}(\%)$ & $16(30.8)$ & $39(48.8)$ & 0.041 \\
Positive EPT, $\mathrm{n}(\%)$ & $14(26.9)$ & $40(50.0)$ & 0.008 \\
Alcohol habit, $\mathrm{n}(\%)$ & $25(48.1)$ & $33(41.3)$ & 0.440 \\
\hline ALT $\mathrm{Al})$ & &
\end{tabular}

ALT: Alanine aminotransferase, AST: Asparatate aminotransferase, BMI: Body mass index, BP: blood pressure, e-GFR: Estimated glomerular filtration rate, EPT: Ethanol patch test, HDL-C: High-density lipoprotein-cholesterol, Hs-CRP: High-sensitivity-C reactive protein, LDL-C: Low-density lipoprotein-cholesterol

a)

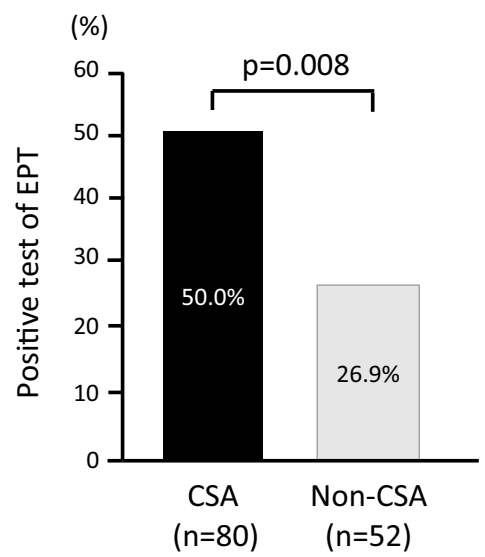

b)

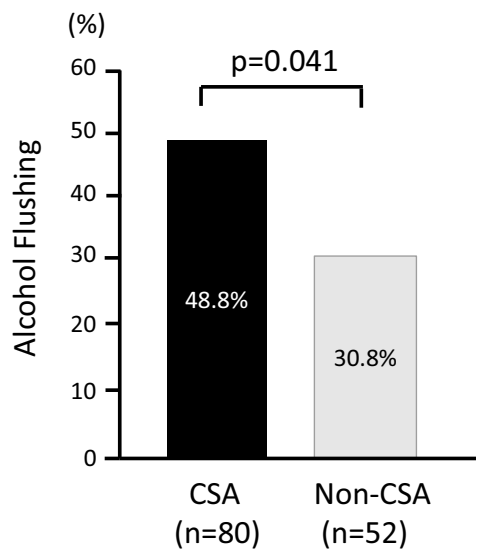

Figure. (a) A comparison of the ethanol patch test and (b) alcohol flushing upon alcohol drinking between CSA patients and non-CSA patients. CSA: coronary spastic angina, EPT; ethanol patch test

nificantly higher $(\mathrm{p}=0.007, \mathrm{p}=0.019$, and $\mathrm{p}=0.007$, respectively) and the plasma levels of HDL cholesterol were significantly lower $(\mathrm{p}=0.035)$ in the CSA group. The rate of positive EPT and alcohol flushing were significantly higher ( $\mathrm{p}=0.008$ and $\mathrm{p}=0.041$, respectively) in the CSA group (Table 1 and Figure). However, there was no significant difference in the proportion of habitual alcohol drinkers between the two groups (Table 1). This may be explained by the fact that alcohol drinking is influenced by social and cultural factors in Japanese society, especially for men. A Spearman's rank correlation analysis revealed that gender (men), smoking, EPT, facial flushing, the plasma levels of HDLcholesterol and the level of uric acid were significantly asso- ciated with CSA $(\mathrm{r}=0.202, \mathrm{p}=0.025, \mathrm{r}=0.194, \mathrm{p}=0.031, \mathrm{r}=$ $0.254, \mathrm{p}=0.005, \mathrm{r}=0.199, \mathrm{p}=0.028, \mathrm{r}=-0.178, \mathrm{p}=0.049$ and $\mathrm{r}=$ $0.266, p=0.003$, respectively). The multiple logistic regression analyses for the predictors of CSA were then performed and included coronary risk factors as independent variables. The variables were sequentially deleted from the models on the basis of theoretical and clinical grounds and weak associations, leaving age, gender, smoking, EPT, uric acid and HDL-cholesterol levels in this model. The analysis revealed that EPT was significant predictors of CSA $(p=$ 0.011) (model 1, Table 2). However, smoking, a well-known risk factor for CSA, did not reach the significant level ( $\mathrm{p}=$ 0.099 ) probably because gender and uric acid were closely 
Table 2. The Results of the Multivariable Logistic Regression Analysis for CSA

\begin{tabular}{lcccccc}
\hline & Coef & Std.Err & $\mathrm{z}$ & $\mathrm{p}>|\mathrm{z}|$ & \multicolumn{2}{c}{$95 \%$ CI } \\
\hline Age & 0.042 & 0.022 & 1.95 & 0.052 & -0.000 & 0.084 \\
Sex (male) & 0.049 & 0.550 & 0.09 & 0.928 & -1.028 & 1.127 \\
EPT (positive) & 1.106 & 0.436 & 2.53 & 0.011 & 0.250 & 1.961 \\
Smoking & 0.869 & 0.527 & 1.65 & 0.099 & -0.164 & 1.903 \\
HDL-C & -0.012 & 0.011 & -1.11 & 0.265 & -0.034 & 0.009 \\
Uric acid & 0.344 & 0.176 & 1.96 & 0.050 & -0.001 & 0.688 \\
cons & -4.254 & 1.972 & -2.16 & 0.031 & -8.119 & -0.390 \\
\hline
\end{tabular}

Number of Observations $=123$, Probability $>X^{2}=0.0007$, Pseudo $R^{2}=0.1422$

_cons: Constant, Coef: Coefficient, Std.Err: Standard Error, CI: Confidential interval, EPT:

Ethanol patch test, HDL-C: High-density lipoprotein-cholesterol

Table 3. The Results of the Multi-variable Logistic Regression Analysis for CSA

\begin{tabular}{lcccccc}
\hline & Coef & Std.Err & $\mathrm{z}$ & $\mathrm{p}>|\mathrm{z}|$ & \multicolumn{2}{c}{$[95 \%$ Conf.interval] } \\
\hline Age & 0.366 & 0.020 & 1.83 & 0.068 & -0.003 & 0.076 \\
EPT (positive) & 1.047 & 0.412 & 2.54 & 0.011 & 0.240 & 0.185 \\
Smoking & 1.026 & 0.424 & 2.42 & 0.016 & 0.195 & 1.857 \\
HDL-C & -0.158 & 0.010 & -1.55 & 0.122 & -0.358 & 0.004 \\
cons & -1.988 & 1.551 & -1.28 & 0.200 & -5.027 & 1.052 \\
\hline Number of Observations=131, Probability $>\mathrm{X}^{2}=0.0007$, Pseudo $\mathrm{R}^{2}=0.1092$ \\
cons: Constant, Coef: Coefficient, Std.Err: Standard Error, CI: Confidential interval, EPT: \\
Ethanol patch test, HDL-C: High-density lipoprotein-cholesterol
\end{tabular}

association with smoking (gender vs. smoking, $\mathrm{r}=0.566, \mathrm{p}<$ 0.0001 and gender vs. uric acid, $r=0.343, \mathrm{p}=0.0001$, respectively). Accordingly, gender and uric acid were excluded from the final analysis (model 2). The analysis then revealed that a positive EPT and smoking were significant predictors of CSA ( $\mathrm{p}=0.011$ and $\mathrm{p}=0.016$, respectively) in this model (model 2, Table 3). The sensitivity and specificity for the detection of CSA were $50.0 \%$ and $73.1 \%$ for EPT and $48.8 \%$ and $69.2 \%$ for alcohol flushing, respectively.

\section{Discussion}

Coronary spasm plays an important role in the pathogenesis of CHD, and its prevalence is especially high in East Asians, including the Japanese population $(1,2,17,18)$. About half of East Asians show rapid and intense alcohol flushing after drinking alcohol in the amount that has no effect on Caucasians (6-8). We and others have shown that CSA could be induced by alcohol ingestion (9-11).

Ethanol (alcohol) is metabolized largely by sequential hepatic oxidation, first to acetaldehyde by ADH and then to acetic acid by ALDH2 (4). About half of East Asians including Japanese have a common ALDH $2 * 2$ mutant phenotype, which results in a deficient enzymatic activity and subjects with this mutant ALDH2 $* 2$ exhibit the characteristic acetaldehyde-induced alcohol flushing when drinking alcohol (6-8). ALDH2 is ubiquitously expressed throughout the body, including the skin (19), and the skin -patch ethanol is converted into acetaldehyde. The EPT has been shown to be a useful marker for the presence of an ALDH $2 * 2$ muta- tion $(8,13,14)$.

In the present study, we performed the EPT, together with the questionnaire on flushing after alcohol ingestion, as a surrogate marker for alcohol intolerance. The results showed that the rate of positive EPT and alcohol flushing after alcohol ingestion were higher in the CSA patients than non-CSA patients, indicating that CSA patients had a significantly association with alcohol intolerance. The multivariable logistic regression analysis revealed that positive EPT was a significant independent predictor of CSA. Smoking, a well-known risk factor for CSA $(1,2)$, did not reach the significant level in this study probably because of its close association with gender and the uric acid level. However, smoking became a significant predictor of CSA when gender and uric acid were excluded from the model in the final analysis.

We have shown that CSA patients have high levels of oxidative stress and endothelial dysfunction (1). ALDH2 plays a key role in the oxidation of various toxic aldehydes as well as acetaldehyde and also protects the myocardium during ischemia (20). In addition, it plays a crucial role in the treatment of myocardial ischemia by converting nitrates to NO and suppressing nitrate tolerance (21). Recent epidemiological studies showed that mutant ALDH2*2 is a significant risk factor for acute myocardial infarction in Japan $(22,23)$ and South Korea $(24)$. It is thus reasonable to speculate that CSA is prevalent in East Asians partly because of its association with mutant ALDH2*2. Further studies are required to elucidate this interesting and important problem. 


\section{Clinical implications}

The results of this study show that alcohol intolerance is associated with CSA in Japanese. Unexpectedly, there was no significant difference in the proportion of habitual alcohol drinkers between the CSA and non-CSA groups. This may be explained by the fact that alcohol drinking is influenced by social and cultural factors in the Japanese society, especially in men. It is therefore recommended that the patients with CSA, particularly those with alcohol intolerance, should abstain from drinking alcohol, as well as smoking, in order to prevent the onset of CSA. However, the sensitivity of EPT for CSA was not high in this study and we do not recommend this test for the screening and/or diagnosis of CSA. It is also to be noted that there may be some patients with CSA whose attack may be relieved by ingestion of small amounts of alcohol because small amounts of alcohol may enhance endothelial nitric oxide activity thereby leading to vasodilatation $(25,26)$.

\section{Limitations}

We did not examine the genetic polymorphisms of ALDH2 in the present study. However, several previous studies have shown that facial flushing after alcohol drinking and/or the EPT are closely associated with ALDH2*2 $(8,13,14)$, and further studies are required. The sensitivity and specificity of the EPT and the questionnaire for the detection of CSA in the present study were not so high probably because the concentration of alcohol in the patch was low compared with those of the previous studies for the detection of the mutant ALDH2*2 $(8,13,14)$. However, the purpose of this study was to examine the association of CSA with alcohol ingestion, not to examine the sensitivity and specificity of the EPT or alcohol flushing after alcohol intake for the detection of CSA.

\section{Conclusion}

We conclude that the EPT and alcohol flushing are significantly associated with CSA in Japanese patients and therefore CSA patients with alcohol flushing should abstain from alcohol drinking as well as smoking.

The authors state that they have no Conflict of Interest (COI).

\section{Funding}

This study was supported in part by the Japan Heart Foundation, Tokyo, and the Japan Vascular Disease Research Foundation, Kyoto, Japan.

\section{References}

1. Yasue H, Nakagawa H, Itoh T, Harada E, Mizuno Y. Coronary artery spasm--clinical features, diagnosis, pathogenesis, and treatment. J Cardiol 51: 2-17, 2008.

2. JCS Joint Working Group. Guidelines for diagnosis and treatment of patients with vasospastic angina (coronary spastic angina). Circ
J 74: 1745-1762, 2010.

3. Pristipino C, Beltrame JF, Finocchiaro ML, et al. Major racial differences in coronary constrictor response between Japanese and Caucasians with recent myocardial infarction. Circulation 101: 1102-1108, 2000.

4. Schukit MA. Ethanol and Methanol. In: Goodman \& Gilman's Pharmacological Basis of Therapeutics. 12th Ed. Brunton L, Chabner B, Knollman B, Eds. McGraw Hill, New York, 2011: 629-647.

5. Takeshita $T$, Yang $X$, Morimoto $K$. Association of the ADH2 genotypes with skin responses after ethanol exposure in Japanese male university students. Alcohol Clin Exp Res 25: 1264-1269, 2001.

6. Oota H, Pakstis AJ, Bonne-Tamir B, et al. The evolution and population genetics of the ALDH2 locus: random genetic drift, selection, and low levels of recombination. Ann Hum Genet 68: 93109, 2004.

7. Li H, Borinskaya S, Yoshimura K, et al. Refined geographic distribution of the oriental ALDH2*504Lys (nee 487Lys) variant. Ann Hum Genet 73: 335-345, 2009.

8. Yokoyama A, Omori T, Yokoyama T. Alcohol and aldehyde dehydrogenase polymorphisms and a new strategy for prevention and screening for cancer in the upper aerodigestive tract in East Asians. Keio J Med 59: 115-130, 2010.

9. Takizawa A, Yasue H, Omote S, et al. Variant angina induced by alcohol ingestion. Am Heart J 107: 25-27, 1984.

10. Seki T, Okayama H, Isoyama $S$, et al. The role of alcohol dehydrogenase 2 and aldehyde dehydrogenase 2 genotypes in alcoholinduced vasospastic angina. Tohoku J Exp Med 187: 311-322, 1999.

11. Morikawa Y, Mizuno Y, Harada E, Kuboyama O, Yoshimura M, Yasue H. Nitrate tolerance as a possible cause of multidrugresistant coronary artery spasm. Int Heart J 51: 211-213, 2010.

12. Nakagawa T, Kajiwara A, Saruwatari J, et al. The combination of mitochondrial low enzyme-activity aldehyde dehydrogenase 2 allele and superoxide dismutase 2 genotypes increases the risk of hypertension in relation to alcohol consumption. Pharmacogenet Genomics 23: 34-37, 2013.

13. Muramatsu T, Higuchi S, Shigemori K, et al. Ethanol patch test--a simple and sensitive method for identifying ALDH phenotype. Alcohol Clin Exp Res 13: 229-231, 1989.

14. Matsuse H, Shimoda T, Fukushima C, et al. Screening for acetaldehyde dehydrogenase 2 genotype in alcohol-induced asthma by using the ethanol patch test. J Allergy Clin Immunol 108: 715$719,2001$.

15. Okumura K, Yasue H, Horio Y, et al. Multivessel coronary spasm in patients with variant angina: a study with intracoronary injection of acetylcholine. Circulation 77: 535-542, 1988.

16. Makino T, Sinnno K, Yokoi H, Matsui C, Morohasi M. Simple ethanol patch test for alcohol tolerance. J New Rem \& Clin 52: 130-135, 2003 (in Japanese).

17. Sueda S, Kohno H, Fukuda H, et al. Clinical impact of selective spasm provocation tests: comparisons between acetylcholine and ergonovine in 1508 examinations. Coron Artery Dis 15: 491-497, 2004.

18. Takagi Y, Yasuda S, Takahashi J, et al; Japanese Coronary Spasm Association. Clinical implications of provocation tests for coronary artery spasm: safety, arrhythmic complications, and prognostic impact: multicentre registry study of the Japanese Coronary Spasm Association. Eur Heart J 34: 258-267, 2013.

19. Goedde HW, Agarwal DP, Harada S. Alcohol metabolizing enzymes: studies of isozymes in human biopsies and cultured fibroblasts. Clin Genet 16: 29-33, 1979.

20. Chen $\mathrm{CH}$, Sun L, Mochly-Rosen D. Mitochondrial aldehyde dehydrogenase and cardiac diseases. Cardiovasc Res 88: 51-57, 2010.

21. Beretta M, Wölkart G, Schernthaner M, et al. Vascular bioactiva- 
tion of nitroglycerin is catalyzed by cytosolic aldehyde dehydrogenase-2. Circ Res 110: 385-393, 2012.

22. Takagi S, Iwai N, Yamauchi R, et al. Aldehyde dehydrogenase 2 gene is a risk factor for myocardial infarction in Japanese men. Hypertens Res 25: 677-681, 2002.

23. Takeuchi F, Yokota M, Yamamoto K, et al. Genome-wide association study of coronary artery disease in the Japanese. Eur J Hum Genet 20: 333-340, 2012.

24. Jo SA, Kim EK, Park MH, et al. A Glu487Lys polymorphism in the gene for mitochondrial aldehyde dehydrogenase 2 is associated with myocardial infarction in elderly Korean men. Clin Chim Acta 382: 43-47, 2007.

25. Krenz M, Korthuis RJ. Moderate ethanol ingestion and cardiovascular protection: from epidemiologic associations to cellular mechanisms. J Mol Cell Cardiol 52: 93-104, 2012.

26. Xue L, Xu F, Meng L, et al. Acetylation-dependent regulation of mitochondrial ALDH2 activation by SIRT3 mediates acute ethanol-induced eNOS activation. FEBS Lett 586: 137-142, 2012.

(C) 2013 The Japanese Society of Internal Medicine http://www.naika.or.jp/imonline/index.html 